\title{
Moiré Fringes Produced by a Point Projection X-ray Microscope
}

\author{
Sanford B. Newman
}

(November 30, 1962)

\begin{abstract}
Moiré fringes produced by soft X-rays passing through crossed gratings of fine wire mesh are demonstrated. Regular systems of bands appearing superimposed on radiomicrographs of oriented cellulosic structures may also be moiré fringes. These fringes could be formed by fibrillate structures acting as crossed diffraction gratings.
\end{abstract}

\section{Introduction}

In the simplest and most familiar case moiré fringes are observed when two parallel, fairly coarse, bar and slit gratings of different pitch are held between the eye and an extended source. When the gratings overlap, the wide bars shown in figure 1a appear. Superimposed graiings of equal pitch will also produce a moiré pattern when given an angular displacement about the normal to the plane of the gratings as shown in figure $1 \mathrm{~b}$. There are, of course, many types of gratings whose fringes cannot be observed in this way, especially if the gratings are of fine pitch. Moreover, moiré patterns containing contributions from both of these basic types are often encountered as well as complexities arising from grating irregularities and angular

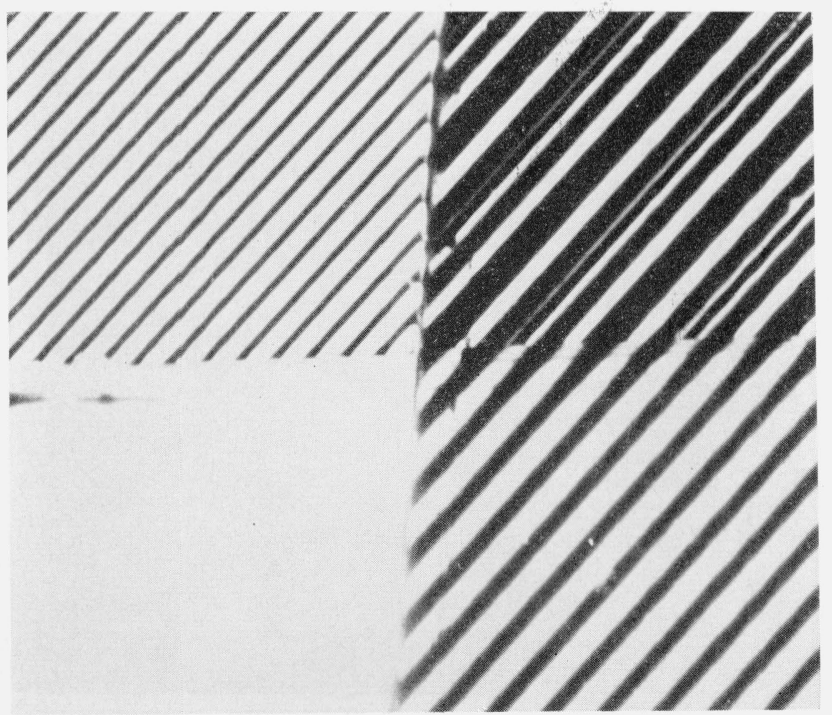

FiguRE 1a. Moire fringes in the upper right quadrant formed by superimposing two Ronchi rulings of different pitch, (upper left and lower right quadrant). displacements other than those mentioned previously. The designation, moiré, is derived from the effect produced by the fabric of the same name when two layers are in contact.

Rayleigh [1] ${ }^{1}$ was probably the first to record observations on a simple arrangement for producing moiré fringes and to indicate the relationship between the spacing of the primary gratings, the spacing of the parallel bars, and the mutual inclination of the two gratings of the set. In recent years these fringes have been studied intensively as part of the diffraction grating development program at the National Physical Laboratory, and characteristics and applications have been presented in great detail by Guild [2]. Electron microscopists have also been

1 Figures in brackets indicate the literature references at the end of this paper.

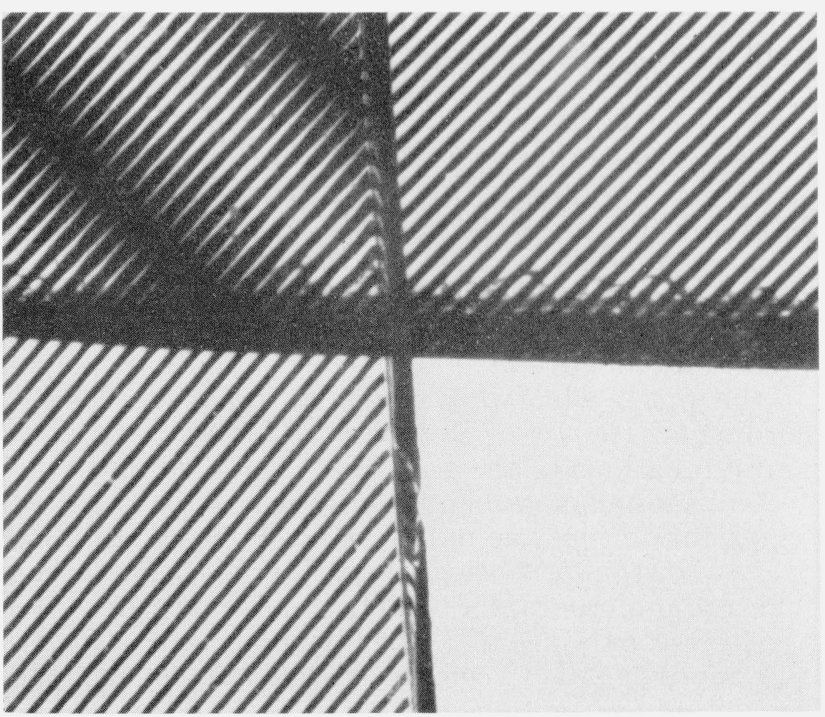

FIGURE 1b. Moiré fringes in the upper left quadrant formed by slight rotation of two Ronchi rulings of the same pitch, (upper right and lower left) about normal to the plane of the gratings. 
able to utilize moiré fringes in the determination of crystal lattice parameters. Overlapping thin crystals act as the gratings to produce fringes, which may be examined and measured at high magnification [3].

Moiré fringes produced by X-rays, however, do not appear to have been noted or utilized in X-ray optics. This paper demonstrates their formation and their similarity to fringes produced by light with the same gracings. The use of such fringes for elucidating structure in point projection X-ray microscopy is also indicated.

\section{Equipment and Materials}

The first practical X-ray point projection microscopes were produced over a decade ago [4] but their availability has been extremely limited until the last few years. All of the observations to be described here were made using electromagnetic microfocus tubes. An instrument designed by Long [5] having only an objective lens was used in the early work. A commercially produced, two-lens, X-ray microscope was used for the subsequent examinations. Both instruments were fitted with copper and aluminum targets and operated at about $15 \mathrm{kv}$ and at not more than $20 \mu \mathrm{a}$.

The fibers were obtained from the collection of the U.S. Department of Agriculture, Bureau of Plant Industry, through the courtesy of Elton G. Nelson.

\section{Observations}

To determine the practicability of forming moiré fringes by means of X-rays, some coarse gratings were observed in the X-ray point projection microscope. A piece of electrodeposited silver fabric having 1000 wires to the inch was folded on itself so that the wires in both directions were nearly parallel. This grating system when examined with a low power light microscope produced the broad moiré pattern of crossed bars shown in figure 2a. The folded fabric was then placed a short distance from the target of a microfocus tube and the projected image of the fabric was photographed. Broad fringes again appear in the image produced by the $\mathrm{X}$-ray beam as seen in figure $2 \mathrm{~b}$. The pattern was visible using either copper or aluminum radiation but the contrast in the radiograph was higher with the latter. Small differences in the pattern are due to displacing of the mesh while it was being transferred for recording of the two images but the general outlines and dimensions of the fringes are quite similar.

A phenomenon which may be related to the moiré fringe formation was noted in specimens of tula istlé (Agave lecheguilla Torr.), a leaf fiber used in cordage. The radiomicrographs of many specimens displayed transverse banding of a surprisingly regular nature. The increment between the bands was usually close to the limit of resolution of the microscope-some 2 or $3 \mu$. Bands were not present uniformly over the entire fiber bundle but would appear in patches often hundreds of square microns in extent. The indi-

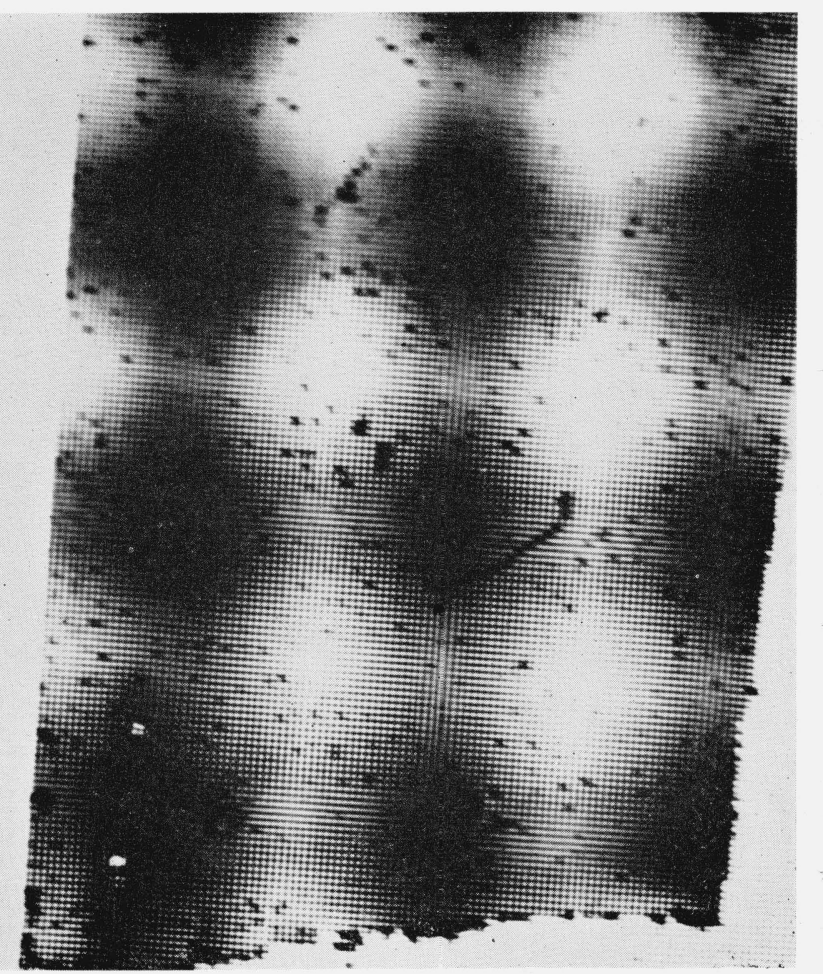

Figure 2a. Silver mesh (1000 wires/in.) folded double.

The wires of the two layers are displaced sufficiently to produce the moiré pattern shown when viewed in white light.

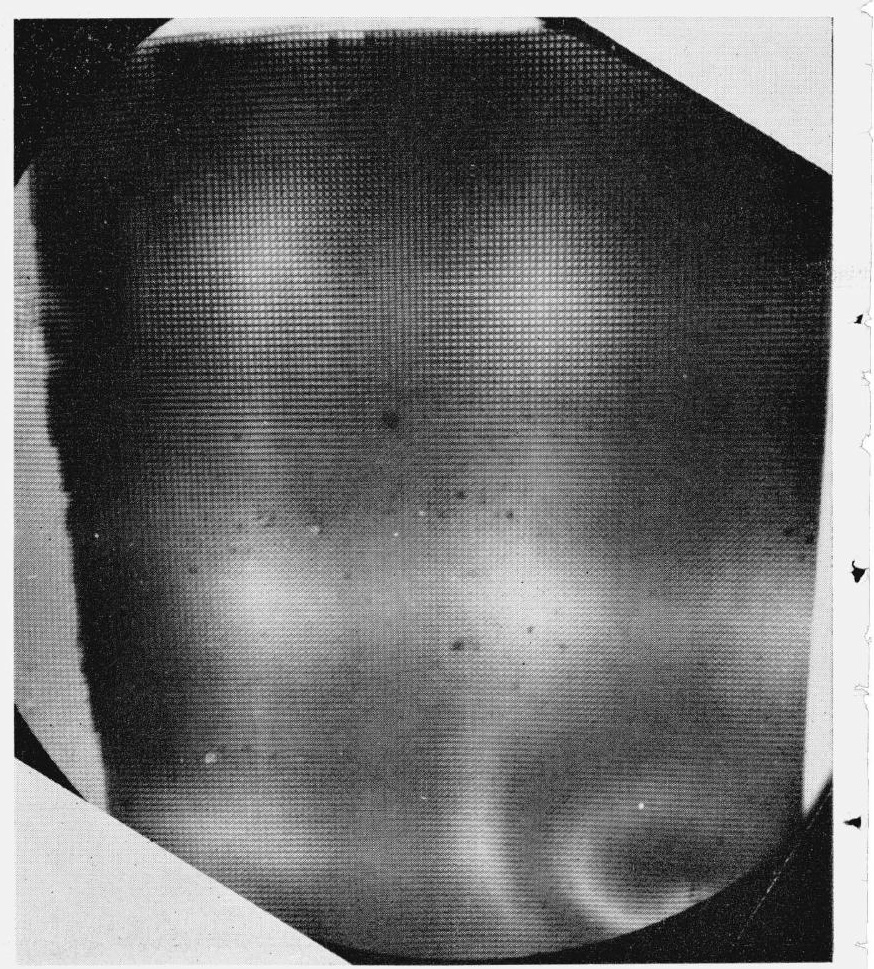

FIgURE 2b. The same specimen as in $2 a$.

The radiograph was made at slightly higher magnification using an aluminum target at $15 \mathrm{kv}$. Slight changes in the pattern are caused mainly by manipulating the specimen in transferring it between instruments. 
vidual bands seemed to have only a rather nebulous relationship with single fibers in the bundle and usually extended over the diameters of several of these components as shown in figures $3 \mathrm{a}$ and $3 \mathrm{~b}$.

The structure of the cell walls of higher plants are well known due to the work of Bailey [6], Kerr [7], and Hock [8], as well as a host of others too numerous to cite here. From these studies it would appear that the only structure which could be correlated with these parallel bars are the spiral windings that form one of the outer layers of the cell wall. This winding consists of a fibrillate thread, a few tenths of a micron in diameter, and make a steep angle with the fiber axis. Since the winding could not be resolved directly by either instrument it might be assumed that windings in opposite sides of fibers or windings on adjacent fibers were acting as gratings to produce the effect.

An alternative source of the bars could be gratings consisting of the fibrillae parallel to the fiber axis vielding rotation moiré. Because of the variables inherent in such a grating system, however, it would be difficult to determine the importance of each of the basic patterns in the fringe formation.

The bars were not exhibited by fibers such as flax (Linum usitatissimum L.) and ramie (Boehmeria nivea (L.) Gaud.) which possess two windings spiralling in different directions. Spacings were observed in a number of agaves, in hemp (Cannabis sativa L.) and in fiber from a luffa sponge (Luffa sp), all of which have only a single peripheral winding.

Staining the fiber structure by precipitating gold or silver did not contribute to the resolution of the bands. As far as can be determined instrumentally the metal is deposited fairly homogeneously in the matrix.

\section{Summary}

Moiré fringes are readily formed by a broad beam of X-rays. These fringes may be an aid in revealing: structure below the projection imaging power of the $\mathrm{X}$-ray microscope.

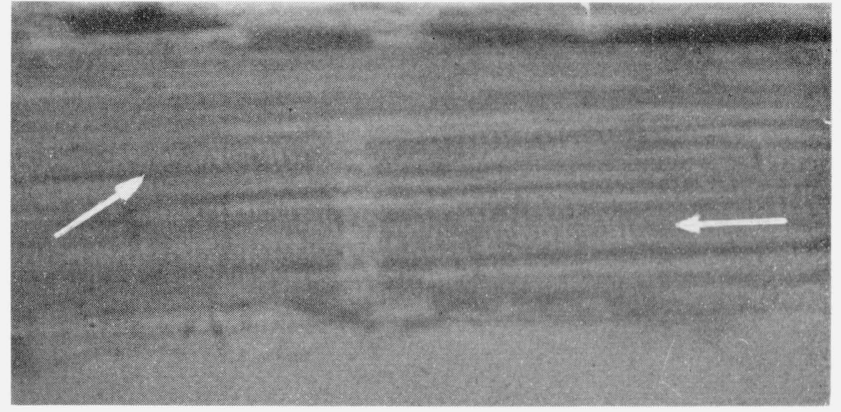

Figure 3a. Transverse bands in microradiographs of the fibers of the fruit of Luffa.

Copper target, $15 \mathrm{kv}$.

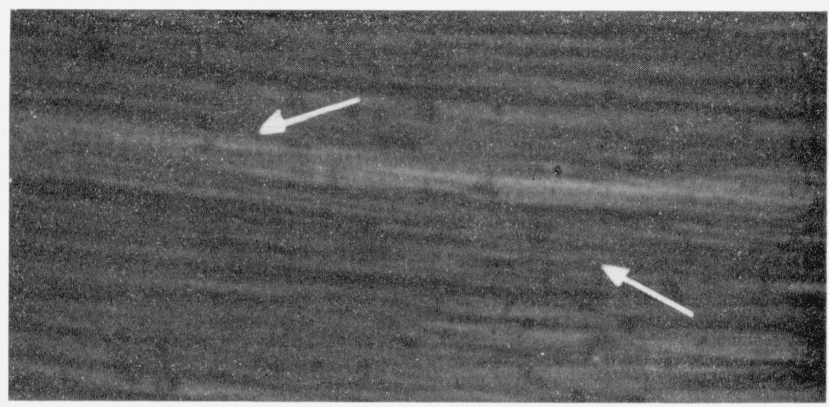

Figure 3b. Transverse bands in microradiograph of leaf fibers of Agave lecheguilla Torr.

Copper target, $15 \mathrm{kv}$.

\section{References}

[1] Lord Rayleigh, Phil. Mag. 47, 81 (1874).

[2] Guild J., Interference Systems of Crossed Diffraction Gratings, Oxford University Press, London (1956).

[3] Menter, J., Advances in Physics 1, 299 (1958).

[4] Cosslett, V. E. and Nixon, W. C., Nature 168, 24 (1951).

[5] Long, J. V. P., Ph. D. Thesis, Cambridge (1959).

[6] Bailey, I. W., Ind. Eng. Chem. 30, 40 (1938).

[7] Kerr, T., Protoplasma 27, 229 (1937).

[8] Hock, C., J. Research NBS 29, 41 (1942).

(Paper 67A2-201) 